\title{
Printed Capacitive Sensors for Contactless Ice Detection in Automotive Liquid Conveyor Pipes
}

\author{
Jakob Happel ${ }^{1}$, Jakob Döring ${ }^{1}$, Karl-Ludwig Krieger ${ }^{1}$, Jonas Deitschun ${ }^{2}$, Dirk Godlinski ${ }^{2}$ \\ ${ }^{1}$ ITEM University Bremen, Otto-Hahn-Allee 1, 28359 Bremen, Germany, \\ jakob.happel@uni-bremen.de, \\ ${ }^{2}$ Fraunhofer Institute for Manufacturing Technology and Advanced Materials IFAM, Wiener Strasse \\ 12, 28359 Bremen, Germany
}

\begin{abstract}
:
This publication focuses on the development of an innovative, low-cost sensor for the detection of ice formation in conveyor pipes of vehicles. The measurement principle is based on the contactless determination of the relative permittivity of a medium by capacitive sensors. In comparison to water, ice has a decreased relaxation frequency through crystallization below the freezing point. Capacitive sensor structures for permittivity measurement were applied on a polymer pipe by functional printing, which can be installed in the conveying systems in the area of the feed lines. Due to its low-cost and universal design, the sensor can be used in all applications where the integrability and costs of an installed sensor system play a major role.
\end{abstract}

Key words: printed capacitive sensors, contactless ice detection, automotive, conveyor pipes

\section{Motivation}

There are various operating fluids in the vehicle, such as windshield washer fluid, cooling fluid, engine oil, servo steering and brake fluid, etc. These are transported by conveyor systems from containers to their destined application sites. Ensuring the operability of the conveyor systems is of great importance since the liquids are necessary for operational and traffic safety.

A loss of vision is a high safety risk in road traffic. Windscreen wiping water is used for cleaning the windshield, often also the headlights and the rear window of a car. At low temperatures, the icing of the liquid is prevented by an alcohol-based antifreeze additive. However, if its content is too low, the solution freezes despite the additive. In particular, the pipes are highly sensitive due to their small diameter. A sensory detection of ice formation in the windshield water pipes with a preventive warning would be useful for the driver.

\section{State of the Art}

Capacitive sensors for the detection of ice formation are already commercially available in stationary systems [1]. In this case, individual sensors are integrated into the roadway and their data is transmitted wirelessly via a weather station located at the roadside, similar to [2].
There are several types of ice formations, which can be measured by capacitance sensors.

In addition to already established ice detection sensors in stationary systems, efforts are also being made to monitor vehicles with the help of suitable sensors. In the aviation industry, safety-relevant, heavily stressed system components, such as wings etc. are continuously checked with an ice detecting system during operation. A method for monitoring water deposition and ice formation on aircraft wings and rotor blades is described in [3]. It is also capable of measuring the thickness of ice layers on the monitored components. Ice detection on large-area structures can be achieved by cost-effective, locally distributed electrodes. Furthermore, these systems can provide information about the effectiveness of used antifreeze by continuously recording and evaluating measurement data during the flight and thus significantly increasing flight safety [4]. Another capacitive sensor for ice detection for aviation applications is presented in [5]. The sensor prevents a sudden blocking of flows in fuel pipes by detecting the icing of water dissolved in kerosene. In this application the coated sensor is installed in the pipe and performs real time measurement.

In accordance with the presented references, mobile condition monitoring systems for ice 
detection were in the past rather used for highpriced applications for economic reasons. By reducing component costs, these systems will become more widely used in the future. In [6], the roadway surface is monitored by capacitive sensors with regard to water and ice presence. The intended field of application is the automotive vehicle due to its cost-effective electrode structure. The sensor electrodes on the vehicle's underbody are excited in different frequency ranges and the system behavior is recorded as a function of the frequency.

\section{Theoretical Background}

Capacitive sensors for ice detection enable a completely contactless measurement, chemically and mechanically isolated from the medium. The basic structure for a capacitive measurement is formed by two conductive electrodes, which build up a capacitor. Conventionally, capacitive sensors consist of two parallel plates, one electrode is for excitation and the other for sensing. The two electrodes are arranged opposite each other and are separated by a dielectric medium with a certain thickness. The electric field between them is evenly distributed in the dielectric. In this arrangement, the stray capacitances can be neglected because of the much larger dimensions of the electrode surface compared to the electrode thickness. In other geometrical arrangements, the field distribution is no longer distributed evenly and the electrical field is not limited to the space between the electrodes. The structure's capacitance depends on the relative permittivity of the medium surrounding the electrodes. Different electrode geometries have application-specific advantages and disadvantages [7] [8] [9], due to their corresponding field distributions.

For the intended application, a concentric, nonmetal pipe with a uniform wall thickness is used. The electrode structures are attached externally by a conductive material, as shown in figure 1 . The spreading electric field penetrates the area in the pipe and thereby also the medium to be monitored. On account of the arrangement, an electric field is also formed outside the pipe which is not negligible.
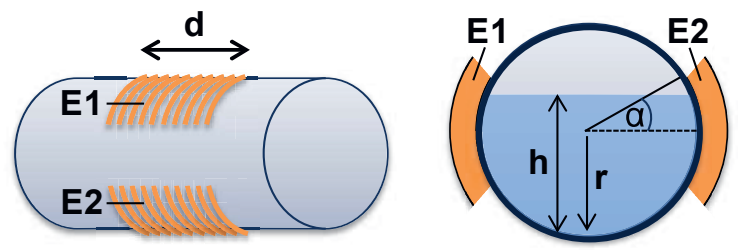

Fig. 1. Conveyor pipe with external electrode structures for capacitive liquid and ice measurement
The capacitive measurement principle is based on the influence of the monitored medium on the electric field between the sensor electrodes. The electric field propagates through the medium and produces a dielectric displacement by the excitation. This dielectric displacement influences the charge stored between the electrodes and thus the capacitance of the sensor. This influence can be used to detect various electrical properties of the medium such as permittivity, conductivity and its distribution [10]. From these measured data material properties such as humidity, temperature and other system parameters can be determined, which are correlated with the electrical properties.

Each dielectric is characterized by its relative permittivity $\varepsilon_{r}$. This is dependent on the ambient pressure, the temperature and the excitation frequency. The dielectric displacement density is described by the following equation:

$$
\vec{D}=\varepsilon_{0} \cdot \varepsilon_{r} \cdot \vec{E}=\varepsilon_{0} \cdot \vec{E}+\vec{P}
$$

The value $E$ represents the electric field and $P$ the dielectric polarization in equation (1). The relative permittivity $\varepsilon_{\mathrm{r}}$ is a measure for polarization, which consequently has an amplifying effect on the dielectric displacement density. For $\varepsilon_{r}$, it follows:

$$
\varepsilon_{r}=1+\frac{1}{\varepsilon_{0}} \cdot \frac{\vec{P}}{\vec{E}}=1+\chi_{e}
$$

Value $X_{e}$ in equation (2) denotes the electric susceptibility. The relative permittivity is a complex value, where $\varepsilon_{r} "$ is the dielectric loss.

$\varepsilon_{r}=\varepsilon_{r}{ }^{\prime}+i \cdot \varepsilon_{r}{ }^{\prime \prime}$

Dielectrics are polarized under the influence of an electric field, i.e. dipoles are induced or permanent dipoles are aligned. In the field of dielectrics it is possible to distinguish between several polarization mechanisms [11]:

- electronic polarization

- atomic polarization

- dipolar/rotational polarization

- space charge polarization

All of the listed polarization mechanisms can be described by their own susceptibility. If a dielectric has several mechanisms, all susceptibilities are added:

$$
\chi_{e}=\chi_{e l}+\chi_{\text {atom }}+\chi_{\text {dip }}+\chi_{s c}
$$




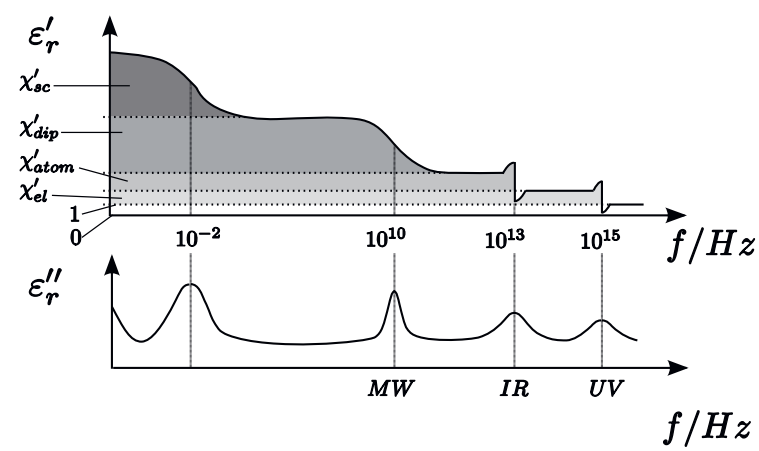

Fig. 2. Frequency response of polarization mechanisms [11]

Resonant polarization mechanisms, like electronic and atomic polarization, are caused by a deflection of charged particles around their center of charge, resulting in an induced dipole. In the case of electronic polarization, the negative electron shell is displaced against the positive atomic nucleus, whereas in the case of atomic polarization, ions of the molecules are displaced elastically within the ionic lattice under the influence of an applied electric field. Due to its low mass, electronic polarization is effective to its cut-off frequency higher than $10^{15}$ $\mathrm{Hz}$, while the atomic polarization cut-off frequency is higher than $10^{13} \mathrm{~Hz}$.

While the electronic and atomic polarization show a resonance behavior, the orientation and space charge polarization have a relaxation behavior. In contrast to resonant polarization mechanisms, in relaxation polarization mechanisms a mechanical reorientation process takes place. While the relaxations have an asymptotic course, the resonances show a characteristic overshoot at the respective resonance frequencies.

The dipolar polarization is very pronounced in the case of permanent dipoles such as water. There are spatially distributed charge centers in a dipolar molecule, which are adjusted along an applied electric field. The Brownian movement causes a countermovement of the alignment depending on the temperature. The cut-off frequency for dipolar polarization is about $10^{9} \mathrm{~Hz}$.

If a dielectric consists of several materials with different specific conductances, charge carrier accumulation occurs in the region of the insulating grain boundaries. This accumulation causes a space charge polarization which has a relatively high permittivity in the range of $\varepsilon_{\mathrm{r}}=10^{5}$ due to a small layer thickness [12]. By means of targeted placement of double layers or locally distributed impurities using the Maxwell-Wagner effect, capacitors with large capacitances can be produced [12]. The space charge polarization is particularly effective in the low frequency range, but under certain conditions it is not negligible even in a frequency range up to $1 \mathrm{MHz}$ [13].

In this publication, the dipolar polarization in particular is used to detect the presence of water and ice and, furthermore, to distinguish between them. There is a characteristic difference between water and ice in the frequency response within frequency range from $10 \mathrm{kHz}$ to $100 \mathrm{kHz}$. While the relaxation frequency $f_{D}$ of the dipolar polarization of unfrozen water is about $10 \mathrm{GHz}$ at $273 \mathrm{~K}$, it shifts to about $3 \mathrm{kHz}$ for ice [14]. This characteristic spectral behavior of $\mathrm{H}_{2} \mathrm{O}$ with the decrease of $\varepsilon^{\prime}(\omega)$ and the peak of $\varepsilon^{\prime \prime}(\omega)$ at a

frequency $f_{D}=\frac{1}{2 \cdot \pi \cdot \tau_{D}}$

is known as the Debye relaxation [14, 15].

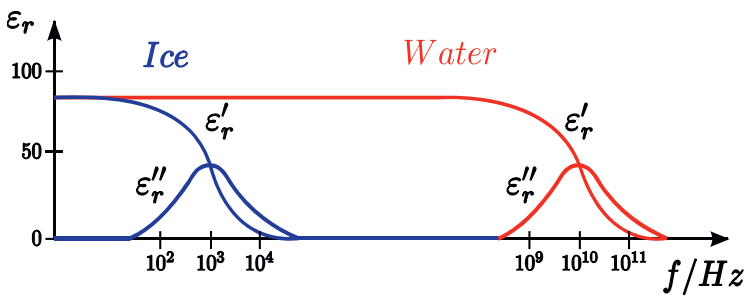

Fig. 3. Water and Ice Dielectric Spectra Scaling at $0^{\circ} \mathrm{C}$ [15]

The dielectric constant $\varepsilon_{\mathrm{r}}$ of $\mathrm{H}_{2} \mathrm{O}$ is dominated by the dipolar polarization because of its large dipole moment. Due to the greater electronegativity of oxygen atoms compared to hydrogen atoms, the electrons are displaced towards the oxygen atom within the molecule so that a negative charge center is formed at this point, while the two hydrogen atoms are positive charge centers. The molecule as a whole remains macroscopically electrically neutral.

Below the crystallization point, water crystallizes in a hexagonal system, and therefore, the anisotropic $\mathrm{H}_{2} \mathrm{O}$ molecules are bounded in a crystal structure. As a result, their mobility is restricted within the structure and the material loses its ability to orient itself in an applied electric field [2]. Depending on the excitation frequency, ice has a characteristic decrease in relative permittivity. While the relative permittivity of water at a measuring frequency of $10^{5} \mathrm{~Hz}$ at $\mathrm{T}=273 \mathrm{~K}$ is about $\varepsilon_{r}^{\prime}=80$, the relative permittivity of ice at $263 \mathrm{~K}$ is approximately $\varepsilon_{r}^{\prime}=4.2$ [5], as shown in figure 4. Based on this characteristic, a capacitive detection of ice formation is possible by measuring the sensor's capacitance at different frequencies. 

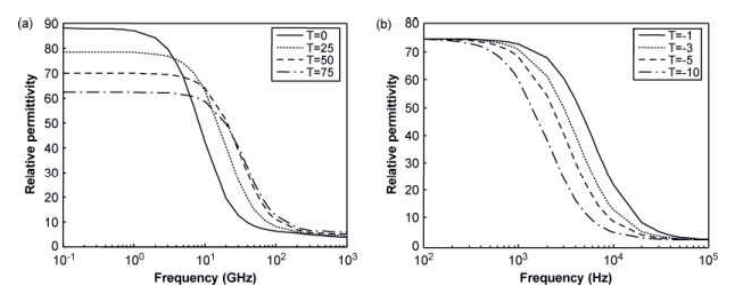

Fig. 4. Comparison between the relative permittivity of water (a) and ice (b) as a function of frequency and temperature [2]

\section{Manufacture of Printed Capacitive Sensors}

In order to meet the high demands in terms of installation space and costs, an innovative concept for function-integrated capacitive measurement has been selected. The goal was to provide cost-effective electrode structures for a wide range of applications, even for costsensitive market segments. For the evaluation of the optimal sensor materials and different electrode layouts, digital printing processes, such as dispensing or micro-jetting, offer time and cost advantages over other manufacturing processes. The geometry of the electrode structures can be flexibly adapted to the requirements of the respective application.

Rotationally symmetrical cylinders made of transparent polyvinyl chloride with outer diameters of 25 and $40 \mathrm{~mm}$, respectively, were used for preparation of samples of functional capacitive transducers. Three different electrode layouts, but each covering the same overall rectangular area of $20 \times 40 \mathrm{~mm}^{2}$ target value were designed, i.e. a complete filled rectangle, a frame and a meander structure, as shown in figure 5 . The effective areas covered with the printed structures were measured optically: $885 \mathrm{~mm}^{2}$ for the complete filled rectangle, $491 \mathrm{~mm}^{2}$ for the frame and $505 \mathrm{~mm}^{2}$ for the meander structure. The area of filled rectangle exceeds the theoretical area of 800 $\mathrm{mm}^{2}$ due to deliquescence of the paste after applying it to the surface. The dimensions of 20 $x 40 \mathrm{~mm}^{2}$ specify the middle of the outer printed line.

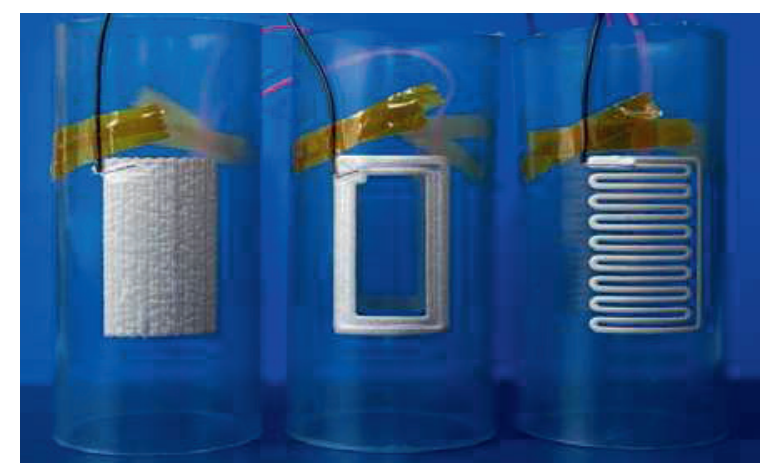

Fig. 5. Electrode layouts, jetted with metal filled conductive paste onto tubes with $40 \mathrm{~mm}$ outer diameter: full, frame, and meander.
After cleaning with isopropyl alcohol, the electrode structures were applied onto the opposite surfaces of the tubes by means of a piezo driven micro-jetting technology (MDS 3000, Vermes Microdispensing $\mathrm{GmbH}$, Otterfing, Germany). The dispensing quantities of this maskless, non-contact system reach from $1 \mathrm{nl}$ to several $\mu \mathrm{l}$ per single drop with media viscosities from $1 \mathrm{mPas}$ up to $2,000,000$ $\mathrm{mPas}$. Smallest typical jetted structure sizes are in the range of 0.1 to $1 \mathrm{~mm}$, depending on nozzle size. In this study, process parameters were chosen as follows: a pressure of 2 bar, a nozzle diameter of $120 \mu \mathrm{m}$, a jetting frequency of $40 \mathrm{~Hz}$ and a linear jetting velocity of $5 \mathrm{~mm} / \mathrm{s}$.

The jetting valve was mounted onto a test bed for functionalizing individual shaped threedimensional customer parts with twodimensional structures [16]. Mechanical base is a CNC milling machine with a collection of tools that can be integrated for scanning, direct writing or other treatments of planar and curved surfaces, respectively. Figure 6 shows the processing of the meander electrode onto a tube with $25 \mathrm{~mm}$ outer diameter mounted in the test bed.

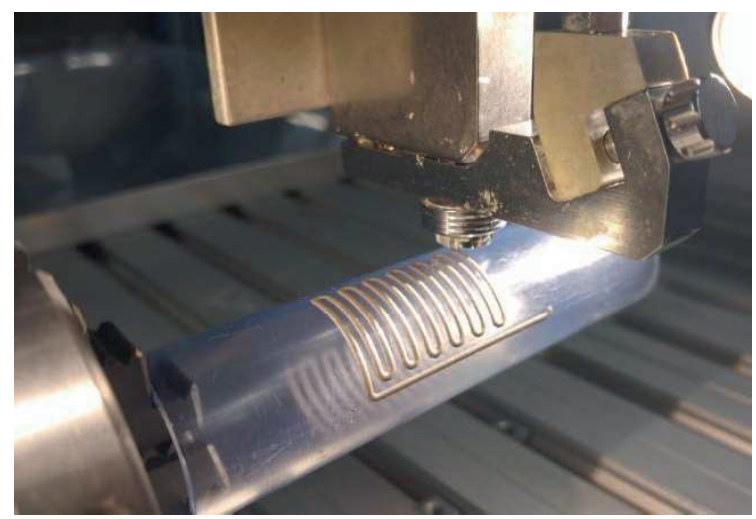

Fig. 6. Micro-jetting of a meander electrode structure onto a tube of $25 \mathrm{~mm}$ outer diameter, mounted in the jetting test bed.

As electrically conductive material a commercial screen printing paste has been used (1901-SB, ESL Europe Ltd., Reading, England). It is a silver-filled, flexible resin material designed for use as a conductor on low-temperature substrates and consists of epoxy resin, butyl diglycol acetate, and silver flakes. The electrical resistance is below $100 \mathrm{~m} \Omega / \square$. For electrical contacting, wires have been placed into one corner of the printed electrodes into the wet paste. The tubes with the printed structures and contacts have been cured together in a furnace at $80{ }^{\circ} \mathrm{C}$ for $45 \mathrm{~min}$. 


\section{Measurement Results}

The measurements were carried out in a Weiss WKL64/70 climatic chamber. It has the dimensions of $470 \mathrm{~mm} \times 345 \mathrm{~mm} \times 400 \mathrm{~mm}$ and the temperature can be varied in the range from $-70{ }^{\circ} \mathrm{C}$ to $+180^{\circ} \mathrm{C}$. The temperature rate of change is indicated at $2.5{ }^{\circ} \mathrm{C} / \mathrm{min}$. The measurement was performed with an AD5933 12-Bit Impedance Converter from Analog Devices. This integrated circuit allows an impedance measurement with a programmable excitation frequency up to $100 \mathrm{kHz}$ and an accuracy of $0.5 \%$. The used thermometer has a waterproof sensor with a measuring range from $-50^{\circ} \mathrm{C}$ to $+110^{\circ} \mathrm{C}$ and an accuracy of $\pm 1{ }^{\circ} \mathrm{C}$.

The validation of the sensing principle was carried out in different measuring series. The following measurements were performed at a measuring frequency of $1 \mathrm{kHz}$ to $100 \mathrm{kHz}$ with a step width of $1000 \mathrm{~Hz}$. The water in the conveyor pipes is thawed at room temperature after freezing in the climatic cabinet at $-30^{\circ} \mathrm{C}$. In this way a thawing of the pipes was modeled by the emitted engine heat after start.

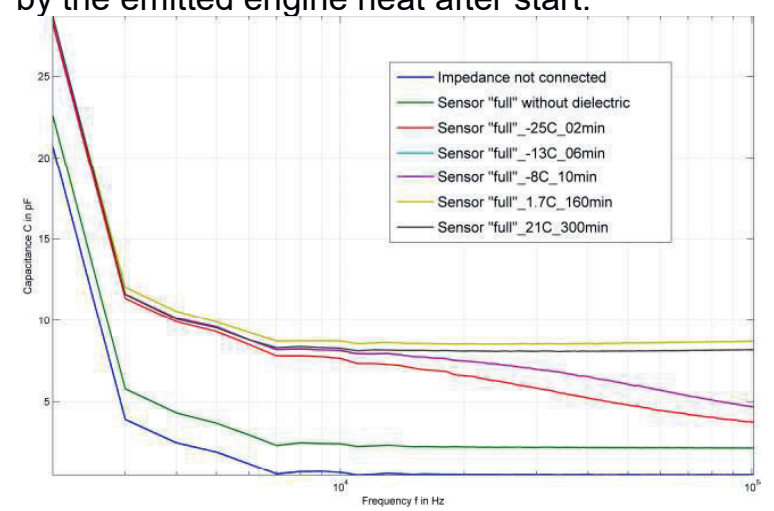

Fig. 7. Measured capacitance over frequency of "full" sensor layout at different temperatures

The measuring system was first calibrated without a connected impedance. A constant offset of $0.5 \mathrm{pF}$ from $10 \mathrm{kHz}$ to $100 \mathrm{kHz}$ is measured for the circuit configuration. The measured value is increased by the capacitance of the sensor without dielectric to $2.2 \mathrm{pF}$. As can be seen from figure 7, the capacitance of the sensor has only a low frequency dependency in the investigated range from $10 \mathrm{kHz}$ to $100 \mathrm{kHz}$. When the sensor is filled, the capacitance increases to about $8 \mathrm{pF}$ at $10 \mathrm{kHz}$, regardless of whether the water is liquid or frozen.

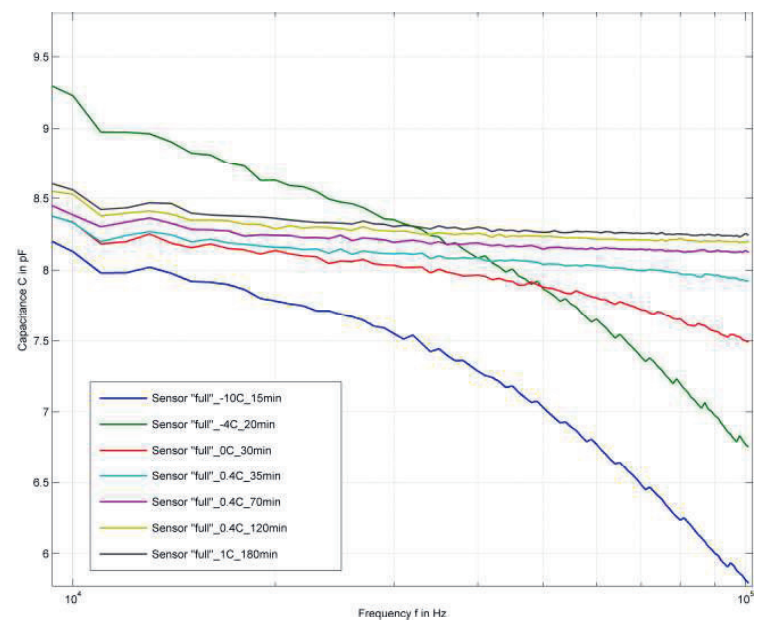

Fig. 8. Measured capacitance for the frequency range from $10 \mathrm{kHz}$ to $100 \mathrm{kHz}$ of "full" sensor layout

The sensor's capacitance can be used to distinguish between the aggregate state of the filled water through a frequency sweep. While the capacitance for ice decreases at higher frequencies, the capacitance for water stays nearly constant. As can be seen from figure 8 , it is advantageous to measure the capacitance for at least two frequencies, for example at $10 \mathrm{kHz}$ and $100 \mathrm{kHz}$.

The thawing process takes place in the pipes from the wall to the center. In the case of partially thawed water with ice core in the center, the frequency dependency decreases with melting of the ice. In case of water with low temperature a higher capacitance value is measured than at higher temperatures. This can be attributed to a higher relative permittivity of liquid water at lower temperatures [2].

As described in the previous chapter, three different sensor layouts were developed to investigate the influence of electrode geometry. The results of this investigation can be seen in figure 9 and table 1.

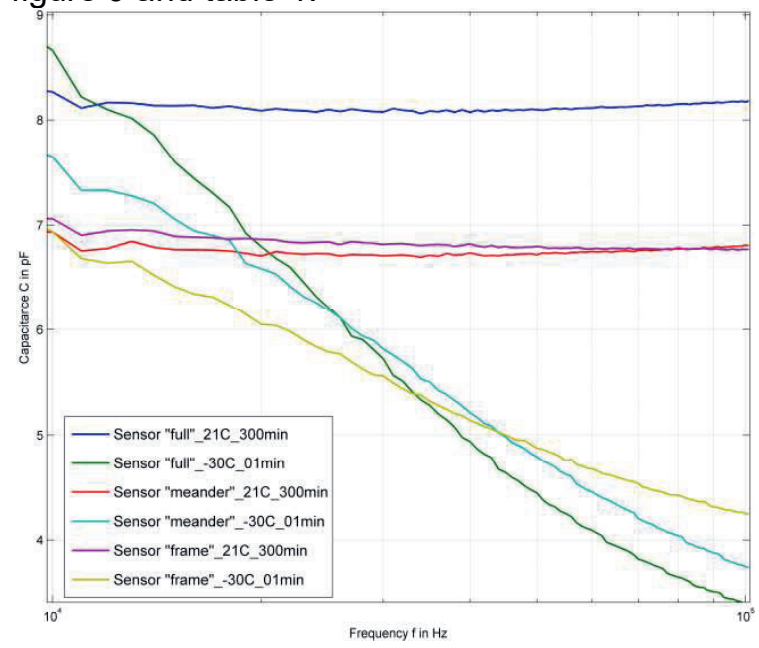

Fig. 9. Measured capacitance over frequency for "full", "meander" and "frame" sensor layouts 
As shown in Table 1, sensor layout "full" has the highest capacitance difference $\Delta \mathrm{C}\left(-30^{\circ} \mathrm{C}\right)$ of $5.26 \mathrm{pF}$ and thus, is best suited for the detection of ice in the pipe. However, the capacitance differences of the other structures with $3.9 \mathrm{pF}$ and $2.69 \mathrm{pF}$ are also clearly measurable, which is sufficient for an ice detection.

Tab. 1: Measured capacitance values for different sensor layouts in $\mathrm{pF}$

\begin{tabular}{|c|c|c|c|}
\hline Sensor Layout & full & meander & frame \\
\hline $\begin{array}{c}\mathrm{C} \text { at } 10 \mathrm{kHz} \\
\left(21^{\circ} \mathrm{C}\right)\end{array}$ & 8.27 & 6.94 & 7.06 \\
\hline $\begin{array}{c}\mathrm{C} \text { at } 100 \mathrm{kHz} \\
\left(21^{\circ} \mathrm{C}\right)\end{array}$ & 8.17 & 6.81 & 6.77 \\
\hline$\Delta \mathrm{C}\left(21^{\circ} \mathrm{C}\right)$ & 0.10 & 0.13 & 0.29 \\
\hline $\begin{array}{c}\mathrm{C} \text { at } 10 \mathrm{kHz} \\
\left(-30^{\circ} \mathrm{C}\right)\end{array}$ & 8.66 & 7.65 & 6.94 \\
\hline $\begin{array}{c}\mathrm{C} \text { at } 100 \mathrm{kHz} \\
\left(-30^{\circ} \mathrm{C}\right)\end{array}$ & 3.4 & 3.75 & 4.25 \\
\hline$\Delta \mathrm{C}\left(-30^{\circ} \mathrm{C}\right)$ & 5.26 & 3.9 & 2.69 \\
\hline
\end{tabular}

\section{Conclusion and Outlook}

The presented measurement results show that a contactless ice detection in automotive liquid conveyor pipes is possible through printed capacitive sensors. For this purpose, three sensors were manufactured in different electrode layouts using the dispensing technology. It has been demonstrated that a distinction between liquid and frozen water can be successfully implemented for all manufactured sensors.

While the maskless micro-jetting or dispensing technology used in this study is ideal for prototyping design or small batch sizes, screen printing could be used for fast and economical production of large batches of functionalised tubes for customer applications using the same functional pastes and layouts.

According to the presented measurements, it is planned to investigate other media in pipes by means of printed sensors. Deionized water and AdBlue are the focus here. It is also planned to investigate the influence of different filling levels for ice detection in automotive sensor pipes.

\section{References}

[1] [Online] http://www.mowic.se/en/ice-warningsystem/

[2] Amedeo Troiano, Eros Pasero, "New system for detecting road ice formation" IEEE Transactions on Instrumentation and Measurement Vol. 60 No. 3, March 2011
[3] Joseph J. Geraldi, et al., " Measuring ice distribution profiles on a surface with attached capacitance electrodes" US Pat. No. US5551288A, September 1996

[4] Seegmiller, H. Lee B., "Ice detector and deicing fluid efficiency monitoring system" US Pat. No. US 5523959, June 1996

[5] H.C. Cho. et al., "Development of a capacitive ice sensor to measure ice growth in real time", Transducers - 2015 18th International Conference on Solid-State Sensors, Actuators and Microsystems, June 2015

[6] K. Cyril Baby, B. George, "A capacitive ice layer detection system suitable for autonomous inspection of runways using ROV ", Robotic and Sensors Environments (ROSE), 2012 IEEE International Symposium, November 2012

[7] Hochstein, Peter. "Capacitive probe for use in a system for remotely measuring the level of fluids" US Pat. No. US5097703 A, March 1992

[8] Maier, Lawrence C. "Apparatus and method for determining liquid levels", US Pat. No. US 4908783 A, March 1990

[9] Tward, Emanuel and Junkins, Philip, "Multicapacitor fluid level sensor", US Pat. No. US4417473 A, November 1983

[10] Alexander V. Mamishev et al., " Interdigital Sensors and Transducers", Proceedings of the IEEE Volume: 92, Issue: 5, pp. 808 - 845, May 2004

[11] Ellen Ivers-Tiffée, Waldemar von Münch, "Werkstoffe der Elektrotechnik", Volume: 10, 2007

[12] Jianjun Liu et al., "Large Dielectric Constant and Maxwell-Wagner relaxation in Bi2 / 3Cu3Ti4O12", Physical Review B 70(14), October 2004

[13] Francesco Pizzitutti, "electrode and interfacial polarization in broadband dielectric spectroscopy measurements", Review of Scientific Instruments Volume 72 Issue 5, April 2001

[14] F. Bordi et al., "Dielectric spectroscopy and conductivity of polyelectrolyte solutions", Journal of Physics Condensed Matter 16(49):R1423, November 2004

[15] V. G. Artemov, A. A. Volkov, "Water and Ice Dielectric Spectra Scaling at $0^{\circ} \mathrm{C}$ ", Ferroelectrics, 466:1, pp. 158-165, May 2014

[16] Godlinski D, Taubenrauch E, Werner C, Wirth I, Zöllmer V, Busse M., "Functional Integration on Three-Dimensional Parts by means of Direct Write Technologies", Fraunhofer Direct Digital Manufacturing Conference (DDMC2012), Berlin (Germany), March 2012 